\title{
Postoperative management following carpal tunnel release surgery: principles of rehabilitation
}

\section{Gregory G. Degnan, M.D.}

Department of Orthopaedic Surgery, University of Virginia Health Sciences Center, Charlottesville, Virginia

The patient with an unsatisfactory result following carpal tunnel release is fortunately the exception rather than the rule. The vast majority of appropriately selected and treated patients will recover uneventfully without the need for extensive therapy. Although uncommon, the dissatisfied patient presents an extremely difficult management problem. Inasmuch as there are a number of factors that may potentially contribute to a poor result, failure to recognize and treat excessive edema and stiffness in the early postoperative period are responsible for a significant percentage of poor results. Patients with a poor result present with three basic signs or symptoms, which may occur in combination or alone. These signs and symptoms include excessive edema, stiffness, and hypersensitivity. If left untreated, these are the patients who will go on to develop persistent median nerve symptoms, stiffness, and possibly even reflex sympathetic dystrophy or sympathetically mediated pain. If identified early and placed in an appropriate therapy program, most of these patients will receive the proper treatment and will ultimately be satisfied with their result. The author presents here a protocol for the postoperative management of the patient who has undergone carpal tunnel release surgery, with emphasis on the recognition and treatment of those patients at risk for a poor result.

Key Words * carpal tunnel syndrome * carpal tunnel release surgery * rehabilitation

Carpal tunnel syndrome is ubiquitous in our society. Any surgeon involved in the practice of peripheral nerve surgery will encounter this problem almost daily. There is enormous controversy and volumes of literature dealing with the issues of the best nonoperative and operative approaches to this problem. These issues will not be addressed in this section. It is my belief that appropriate patient selection and adequate nerve decompression are more important than the type of incision or instrument used. The literature would seem to bear this out, as the results with various methods are all predictably good with high success rates.

This section will focus on something that has received considerably less attention--the general approach to postoperative carpal tunnel syndrome and the early identification and treatment of those patients at risk for a poor outcome. Tubiana[4] has stated that "... despite its high incidence and its reputation for simplicity and efficiency, carpal tunnel release does not invariably produce good results, and dissatisfied patients are not infrequently encountered." Poor outcomes result from a number of factors including inaccurate diagnoses, iatrogenic surgical complications, associated neuropathies, and all too often, the 
secondary gains of litigation and Workers' Compensation.[2,3] I believe that when these variables are eliminated, poor results are often the product of unrecognized and inadequately treated edema of the hand in the early postoperative period.

Edema is the inevitable consequence of injury (traumatic or surgical) to the hand. Postoperative immobilization further contributes to edema by limiting the patient's ability to compress the dorsal veins and produce retrograde venous and lymphatic flow. In the acute phase, edema can delay healing and cause pain and stiffness in the hand. Chronic edema leads to soft-tissue fibrosis and fixation of tissue planes that normally glide in relation to each other. Failure to control edema and maintain normal motion and soft-tissue planes leads to fibrosis, stiffness, and hypersensitivity.[1]

\section{MANAGEMENT PRINCIPLES AFTER CARPAL TUNNEL RELEASE SURGERY}

At our institution, postoperative management actually begins at the preoperative visit. We believe that patient education about the importance of edema control is critical. At the preoperative visit the patient is told that postoperative elevation of the hand and small joint motion are crucial to attaining a good result. The patient receives instruction in elevating the extremity and "fisting" techniques. We emphasize to the patient that wiggling the fingers is inadequate. We demonstrate the flattening of the dorsal veins with full fisting and discuss the importance of allowing the nerves and tendons to glide with full motion. The practice of overhead fisting incorporates the principles of elevation, active digital motion, and shoulder motion. These techniques are the mainstay of our immediate postoperative program.

In the early postoperative period pain control and early follow-up evaluation are crucial. Many patients do not require pain medication following carpal tunnel release surgery; however, withholding pain medication during these first few days can deter the patient from aggressively moving the hand. This leads to increased edema and potential disaster. We routinely see our patients for their first follow-up visit at 4 to 5 days postoperatively. At this point we assess the patient's wound and the degree of swelling and stiffness. The bulky postoperative dressing is removed and replaced with a light-weight removable splint. Most important, we use this early visit to identify which patients will do well with a home therapy program and which patients will require aggressive supervised therapy. Those patients who have minimal edema and good range of motion are instructed in a home program that will control edema and facilitate increased range of motion. We encourage them to expand their activities of daily living as tolerated. The home treatment program consists of continued overhead fisting and string wrapping exercises. String wrapping is easy, inexpensive, and well tolerated by the patients. A piece of soft cord is used and the digits and hand are wrapped from the distal to proximal sides. The hand is then elevated for 5 minutes, the string is removed, and the patient performs full fisting exercises.

We routinely reevaluate these patients at 2 to 3 weeks postsurgery. If they continue to do well we instruct them in scar massage and desensitization techniques. At this point we also have them begin a formal strengthening program. Play Dough, Silly Putty, or wet wash cloths are inexpensive and readily available resources for grip and pinch strengthening. By 3 to 6 weeks postoperatively these patients are usually back to full activity.

Patients who present for their first postoperative visit with significant stiffness and edema require much more aggressive management. These patients are sent to a hand therapist for instruction in a home program and for supervised therapy until edema is under control. The home treatment program includes elevation of the hand, retrograde massage, coban wrapping (or string wrapping), and an Isotoner glove. The patient is also instructed in active range of motion and tendon-gliding techniques. Edema control and 
increasing range of motion are the main foci of therapy for the first 3 weeks. At 2 to 3 weeks the foci are shifted to scar management and strengthening. Elastomer patches and scar massage are the mainstays of scar management during this phase of treatment. Fluidotherapy may also help with scar sensitivity; however, it should be used with caution in the face of persistent edema. During this second phase of rehabilitation some patients will be restricted by persistent pain and hypersensitivity. A transcutaneous electrical nerve stimulator unit can be extremely helpful with these difficult patients. It should be emphasized that any time an early reflex sympathetic dystrophy is suspected, the surgeon should take an aggressive approach. Immediate appropriate therapy and early consultation with a pain management specialist are usually successful in turning these patients around before a full-blown dystrophy develops.

\section{DISCUSSION}

Carpal tunnel release surgery, performed in properly selected patients is a minimally morbid, predictably successful operation. We have all experienced the frustration of treating patients who have not achieved the expected result. In these situations there are often factors that are beyond our control; however, suitable follow-up evaluation and the application of appropriate therapy are two critical factors that we can and should control.

I would like to emphasize that I believe that the vast majority of patients who undergo carpal tunnel release surgery never require formal therapy. Pre- and postoperative patient education concerning the self-management of the extremity is usually successful in restoring normal function. This minimizes the costs to the patient and health care system.

There is, however, a small subset of patients who will not do well with self-management. These patients present early on with swollen, stiff, and painful hands. Early follow up is critical in identifying these patients before edema becomes chronic and fibrosis and small joint contracture become established. When these patients are identified they should be treated aggressively by using a combination of supervised and home therapy programs. Early control of edema and restoration of normal range of motion are paramount.

\section{References}

1. Baxter-Petralia PL: Therapist's management of carpal tunnel syndrome, in Hunter JM, Schneider LH, Makin EJ, et al (eds): Rehabilitation of the Hand: Surgery and Therapy. Philadelphia: CV Mosby, 1990

2. Eason SY, Belsole RJ, Greene TL: Carpal tunnel release: analysis of suboptimal results. J Hand Surg (Br) 10:365-369, 1985

3. Higgs PE, Edwards D, Martin DS, et al: Carpal tunnel surgery outcomes in workers: effects of workers' compensation status. J Hand Surg (Am) 20:354-360, 1995

4. Tubiana R: Carpal tunnel syndrome: some views on its management. Chir Main Memb Svp 9:325-330, 1990

Manuscript received May 21, 1997.

Accepted in final form June 10, 1997. 
Address reprint requests to: Gregory G. Degnan, M.D., Department of Orthopaedic Surgery, University of Virginia Health Sciences Center, Box 159, Charlottesville, Virginia 22908. email:

GD4c@avery.med.virginia.edu. 